\title{
CONTROL PERCEPTIONS AND CONTROL APPRAISAL: RELATION TO MEASURES OF SUBJECTIVE WELL-BEING
}

\author{
FRANCES E. OWUSU-ANSAH \\ Faculty of Medicine, Department of Behavioural Sciences, University Post Office \\ Kwame Nkrumah University of Science and Technology, Kumasi, Ghana
}

Author for Correspondence: FrancesE.Owusu-Ansah,E-mail:feoansah@yahoo.com

Conflict of Interest: None declared

\begin{abstract}
SUMMARY
Objectives: To investigate the relationship between control variables and measures of subjective wellbeing. The primary question asked was "who is happy and well, and why?"
\end{abstract}

Design: A questionnaire survey and the data was analyzed by examining bivariate relationships between variables of interest.

Participants: Two hundred and fifty six (256) adults within the age range of $18-88$ years. They were mainly undergraduate psychology students from South-Eastern part of the United States and solicited in a manner consistent with the Institutional Review Board of the Florida State University. Elderly persons from community groups (e.g. churches, social organizations) were solicited through their appropriate leaders and announcements.

Results: The mean age of the participants was 32 years. Findings indicated significant relationship between control measures, indices of wellbeing, and individual's satisfaction with the quality of their lives. Significant correlations were between 0.177 and 0.469 at the 0.01 level (2-tailed). Having control in the areas of social support, self-acceptance, positive relationships, purpose in life, and personal growth were judged important.

Conclusion: The findings of this study suggest that essential 'ingredients' for psychological and subjective well-being include enjoyment of good health, some financial stability, meaningful ties with others, a sense of purpose and direction in life, ability to manage complex life demands, a healthy self-acceptance and commitment to personal development. Implications for clinical utility and future research are discussed.

Keywords: Perceptions of control, control appraisal, subjective well-being, satisfaction with life, selfacceptance, positive relationships, and mastery

\section{INTRODUCTION}

The perceived control literature consistently shows that being in control over events plays a critical role in people's psychological and physical well being. ${ }^{1,2} \mathrm{~A}$ high perception of control has been associated with positive effects on different areas of life such as academic achievement ${ }^{3}$, positive self-concept ${ }^{4}$, attenuation of job related stress $^{5}$ and healthy psychological functioning. ${ }^{2,6,7,8}$ Perceived control has positive effects on adjustment and coping with varied physical health problems such as AIDS $^{6}$, cancer, and Parkinson disease. ${ }^{8}$

The importance of perceived control lies in its effects on subjective well-being. ${ }^{4}$ Perceived control enhances an individual's assessment of the controllability of a situation to elicit the necessary coping strategy. ${ }^{1,2,9,10} \mathrm{~A}$ situation can be appraised as threatening if one perceives a lack of control over the situation and challenging if the individual perceives the stressor as controllable. A situation that is appraised as threatening is likely to be stressful because the person sees the confronting demands as exceeding his or her coping abilities and this perception creates stress with adverse effect on subjective well-being. ${ }^{11}$

Subjective well-being has been described as the absence of undesirable negative emotional states, such as depression, and the experience of more positive moods and emotions. ${ }^{12}$ It also refers to the way people perceive and evaluate their lives ${ }^{13}$. Research on subjective well-being is growing rapidly not only because of its important socio-economic implications but also because it enhances our understanding of why some people seem happier than others.

As a construct, subjective well-being can be globally assessed or examined in detail from its hallmarks or specific indices. The Diener, Emmons, Larsen \& Griffin Satisfaction with Life Scale (SWLS) is widely used for global assessment ${ }^{14}$ of subjective well-being. When globally assessed, the focus is on the more enduring and pervasive feelings of well-being, not just momentary moods. ${ }^{11}$ Individuals, as it were, take a mental survey of their lives in general and evaluate how happy or how satisfied they are with their quality of life as a whole. In contrast, Ryff ${ }^{15,16}$ outlines 
domain specific measures of subjective well-being that evaluates areas of functioning such as relationships, social support, and personal growth which are essential for mental health (see Appendix A). It appears that domain specific indices, such as Ryff's would be better indicators of psychological health as well as subjective well-being because not all happy persons are necessarily psychologically healthy. A delusional person can be perfectly satisfied and happy with his life although the mental health of the person is questionable. Similarly, some people who are socially functional and even productive are not necessarily happy. Thus although understanding subjective wellbeing is important, it is neither sufficient nor identical with psychological health. ${ }^{13}$ Ryff's measures can provide insights into the essential components of subjective well-being which I believe includes psychological health or wellness. A psychologically healthy person can be expected to have positive attitude towards the self and others, give and receive support, have the ability to build trusting relationships, and committed to continued self development. ${ }^{16}$

Whereas perceived control has been extensively researched, the value or importance attached to it, which is sometimes referred to as control appraisal, has been relatively ignored ${ }^{11}$. This dimension of perceived control is important because of its potential adverse effect on subjective well- being. Lack of control over subjectively important events would surely affect one's life more than lack of control over events considered unimportant. ${ }^{17}$ Therefore, it is essential that the appraisal of the value dimension of perceived control is investigated so that the impact of perceived control on subjective well-being can be better understood.

Control can be secondary or primary. ${ }^{4}$ Secondary control is accommodative and involves attempts to fit in with the environment. Primary control involves attempts to change the environment so that it fits with an individual's needs and desires ${ }^{1}$. It is assimilative and outwardly directed because the individual engages in "whatever means" are necessary, direct or indirect, to persist in the pursuit of desired goals. Tenacious engagement of the environment through one's own efforts is selective primary control and the use of secondary resources to alter the environment and attain goals is called compensatory primarycontrol. ${ }^{18}$ Selective and compensatory controls are together referred to as primary control. Likewise, for clarity in this study, both Diener's SWLS and Ryff's indices are together referred to as subjective well-being.

This study investigated the relationship of perceived control and control appraisal to Ryff's indices and general satisfaction with life. Specifically, three questions were posed. First, what is the relationship between individual's perceived control, their satisfaction with life, and Ryff's indices of subjective well-being?

Second, what is the relationship of control appraisal (value dimension of perceived control) to satisfaction with life and Ryff's indices of subjective well-being? Third, what is the relationship between satisfaction with life and Ryff's indices of subjective well-being? On the basis of the preceding literature and what is known about control and subjective well-being, the following predictions were made.

\section{Hypotheses}

Perceived control is expected to be positively related to both satisfaction with life and Ryff's indices of subjective well-being. If indeed a perception of control is important to well-being, then it is likely to be valued. If so, then control appraisal (value dimension of perceived control) can be expected to have positive correlations with satisfaction with life and Ryff's indices of subjective well-being.

Ryff's indices of well-being and Diener"s Satisfaction with Life Scale (SWLS) are both measures of subjective well-being and therefore they can be expected to be positively related. Though the former is more specific and the latter is a global assessment, the nature and strength of the relationship between them will elucidate our understanding of subjective wellbeing construct.

\section{METHODS}

\section{Subjects}

Participants consisted of 256 adults from South-Eastern United States who were mainly undergraduate psychology students. A few others were solicited from church and social organizations.

\section{Procedure}

Following the approval of the study by the Florida State University Institutional Review Board, undergraduate student participants were solicited in a manner consistent with the process of conducting research in the Florida State University Department of Psychology. Provision of signup sheets enabled participants to choose convenient times for participation. Persons from churches and social organizations were solicited through their appropriate group leaders and announcements.

All items, including demographic information, were combined to create a single packet of questionnaire. Completion of questionnaires was preceded by provision of information about the study, confidentiality, and consent forms read and signed. 
Particular effort was made to ensure consistency in the collection of data through the provision of same stepby-step instruction for the administration of questionnaires for all who assisted in the collection of data.

\section{Measures}

\section{Ryff's well-being scale}

The scale consists of items assessing various domains of functioning such as health, social support, and financial satisfaction, sense of purpose, growth, autonomy, mastery and positive relationships ( See Appendix A). It is rated on a 5-point scale ranging from strongly disagree (1) to strongly agree (5). Scores are averaged and higher scores indicate higher satisfaction in a given domain. This scale has good convergence with other well-being scales assessing similar constructs and consistently adequate reliability 15,19

\section{Satisfaction with Life Scale (SWLS)}

The Diener, Emmons, Larsen \& Griffin ${ }^{14}$ Satisfaction with Life Scale was used as a more global measure of subjective well-being to augment that of Ryff (See Appendix C). The scale consists of 5 statements assessing overall satisfaction with life and rated on a 7point scale from strongly disagree (1) to strongly agree (7). Higher scores indicate higher satisfaction with the quality of one's life in general. The SWLS has good internal consistency and stability with desirable convergent and discriminate validity. ${ }^{14}$

\section{Control Items}

These were adapted from the Brandstadter and Renner's ${ }^{1}$ Tenacious Goal Pursuit \& Flexible Goal Adjustment Scales, the Peng ${ }^{20}$ and Wrosch, Hechausen \& Lachman Scales. $^{21}$ On a given control item, participants rated their perception and appraisal (value or importance) of the control item on a 7-point scale ranging from very bad (1) to very good (7) and not important (1) to very important (7). (See Appendix B for Control Items) A mean score was computed with higher scores indicating higher perceptions of control and higher control appraisal (importance). Internal consistency reliabilities on the control items were acceptable. Mean ranged from 4.67 to 5.15 with .97 standard deviation and coefficient alpha of .72 and .60 for selective primary control and compensatory primary control respectively.

\section{RESULTS}

There were 192 women (72.5\%) and 73 men (27.5\%) within the age range of $18-88$ with a mean age of 32.5 years. Most were single or never married (73.6\%), in a committed relationship (21.2\%), divorced/separated $(31.1 \%)$ or widowed $(2.0 \%)$. Several had completed college $(66.4 \%)$ or were still in college $(24.2 \%)$ and a few High School graduates (9.4\%). Participants were ethnically diverse with Caucasian and AfricanAmerican groups forming the majority (51.7\%) and (33.3\% respectively); and a few Ghanaian (4.9\%), Hispanic (4.9\%) and Asian (3.4\%) groups.

Data was analyzed by examining bivariate relationship between variables. The research question investigated the relationship of perception and appraisal of control to measures of subjective well-being. As predicted in the first hypothesis, perceived control was positively and significantly correlated with life satisfaction (Table 1). Perceived control was also positively related to other measures of subjective well-being such as physical health, social support, self-acceptance, purpose in life, autonomy, mastery, growth and positive relationships. Financial satisfaction was not related to perception of control in a positive or significant way.

Table 1 Correlation among Perception and Appraisal of Control and Measures of Subjective Well-Being

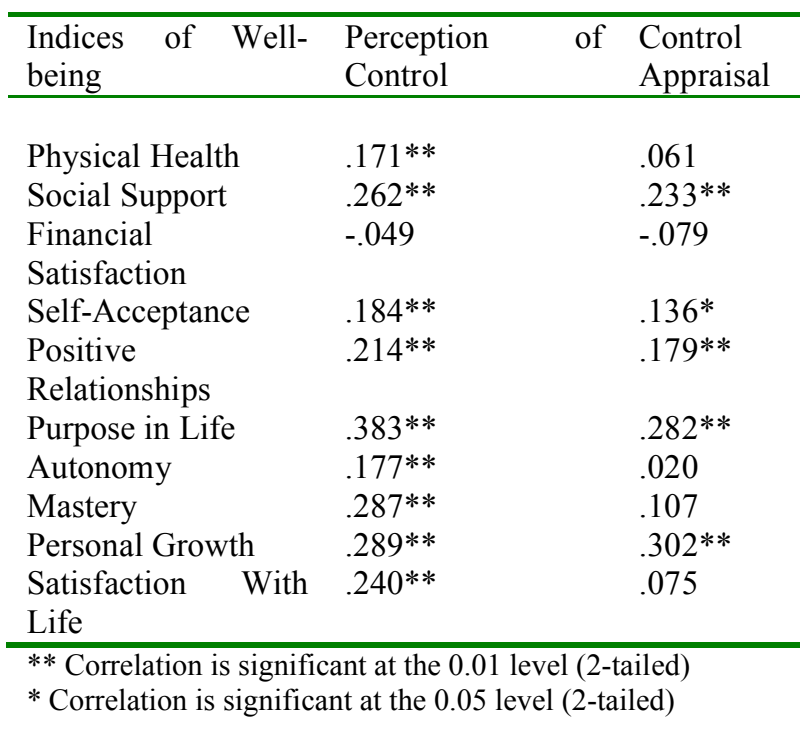

A perception of control was positively and significantly appraised as important in social support, positive relationships, self-acceptance, a sense of purpose in life, and growth suggesting that participants valued their perception of control over these areas of their lives.

However, contrary to prediction, control appraisal was not significantly related to life satisfaction and, as indicated, it was not positively correlated with all of Ryff's indices of well-being. The third hypothesis was mostly confirmed. With the exception of the autonomy measure, there were positive and significant relationships between satisfaction with life and Ryff's 
indices of well-being affirming the similarity of the constructs (Table 2)

Table 2 Correlations between Satisfaction with Life and Ryff's Indices of Subjective Well-Being.

\begin{tabular}{ll}
\hline Ryff's indices of well-being & Satisfaction With Life \\
\hline Health & $.224^{* *}$ \\
Social Support & $.469^{* *}$ \\
Financial satisfaction & $.463^{* *}$ \\
Self Acceptance & $.599^{* *}$ \\
Purpose in Life & $.276^{* *}$ \\
Autonomy & .101 \\
Mastery & $.458^{* *}$ \\
Positive Relationships & $.381^{* *}$ \\
Personal Growth & $.158^{* *}$ \\
\hline
\end{tabular}

** Correlation is significant at the 0.01 level (2-tailed)

\section{DISCUSSION}

Cultural context largely determines how and what people consider when evaluating the quality of their lives. 16,22,23 Persons from collectivists and individualist cultures differ in the way they view themselves and their lives. ${ }^{24}$ The lack of cultural orientation measure in this study can be considered a limitation.

This limitation notwithstanding, the study provides insights into what contributes to individual's evaluation of their lives as satisfying or dissatisfying. Previously researchers have asked the question "who is happy and why" "2 ? Now we can expand that question to include the understanding of not just who is happy but "who is happy and well, and why" because as noted earlier," happiness" is not synonymous with "wellness". ${ }^{16}$

Many will agree that psychological well-being is not just the absence of sickness such as anxiety, depression, and other forms of mental problems but an emphasis on positive characteristics of growth and development. Ryff ${ }^{16}$ argues that "wellness" is not synonymous with happiness" although being "well" can contribute to one's happiness. According to Ryff, psychological wellness or well-being includes essentials such as positive attitude toward self and one's past (self-acceptance), possession of goals and objectives that give meaning to life (purpose), caring and trusting ties with others (positive relationships), continued development and self-realization (growth) and the ability to manage complex demands of daily life (mastery) and act from own convictions (autonomy).

Subjective well-being, like any other construct, is better understood by examining its correlates and disaggregating its various components. Although this study uses a less sophisticated method of analysis (correlations), important insights on subjective wellbeing can be gleaned from the findings. The Satisfaction With Life Scale (SWLS) ${ }^{14}$ showed good convergence with Ryff's measures of well-being suggesting similarity in what both scales measure. The utility of this information lies in the observation that increases in individual's assessment of the quality of their lives as generally happy were accompanied by similar positive evaluations in other life domains such as physical health, relationships, social support, selfacceptance, purpose in life, personal growth and a feeling of mastery or competence. Thus the use of both SWLS and Ryff's measures not only serve to augment the assessment and our understanding of subjective well-being, the findings suggest that subjective wellbeing is an evaluative judgment with varied components that go beyond hedonism. ${ }^{25}$

This view is consistent with findings of previous research that call attention to other factors contributing to individual's sense of well-being. Social support, for example, is not only significantly associated with subjective well-being but it is also related to positive effect, better coping response, reduction of depressive symptoms and negative affect. ${ }^{26-30}$ Adolescents with high levels of social support network report better psychological health and adjustment because social support network is a key intervening variable in physical and mental health. ${ }^{26}$ Similarly, self acceptance, a sense of purpose in life, and personal growth are importantly related to subjective well-being particularly during the crucial stage of human development called adolescence. ${ }^{31,16}$ The substantial influence of these factors on subjective well-being, especially physical health and social support, continues through later adulthood. ${ }^{28}$

The findings also attest to the important and well established relationship of perceived control to subjective well-being. ${ }^{7,9,11}$ Increases in perception of control were associated with better physical health, greater social support, self-acceptance, a sense of purpose in life, autonomy, mastery, growth and positive relationships and a general sense of satisfaction with the quality of one's life. More importantly, the appraisal of a perception of control in the areas of social support, positive relationships, purpose, self-acceptance and personal growth was significant. In other words, participants perceived that having control in these areas of their lives was very important to them.

There was no significant relationship between control appraisal and the areas of autonomy, mastery, and physical health. Similarly, being autonomous was not 
significantly related to satisfaction with life. Could it be that, with age and experience, people learn that tenaciously holding unto a perception of control over one's physical health, financial status, autonomy, and mastery is an exercise in futility? Or could the findings be reflective of this sample and their held values?

A replication of the study with larger and less diverse sample can help elucidate this finding.

Contrary to previous findings increases in financial satisfaction were accompanied by increases in satisfaction with life.

Previous work suggest that rapid increases in income lowered subjective well-being; suggesting that with increasing income expectations can rise too quickly to be realised ${ }^{24}$ thus breeding dissatisfaction, depression, anxiety, isolation and alienation. ${ }^{11}$ It appears that having enough money for basic needs without the excesses is better for subjective well-being. ${ }^{12,13}$

\section{CONCLUSION}

The findings of this study suggest that essential 'ingredients' for psychological or subjective well-being include enjoyment of good health, some financial stability, meaningful ties with others in the form of relationships and social support, a sense of purpose and direction in life, ability to manage complex life demands, a commitment to personal development of control in these areas is equally important for wellbeing; especially if control in that domain is deemed important. If these variables indeed help people live more fulfilled lives, then serious consideration and investment of time, effort, and research in these areas are worthwhile.

Clearly subjective well-being is multifaceted. Some aspects of the construct have been investigated and understood and others are not. For instance while previous research has clearly established the positive impact of educational attainment, occupational status, and social class differences on subjective well-being 27,28 the impact of religion and spirituality is less studied and psychologists disagree on the issue. ${ }^{27}$ Carl Jung believed that people fell ill when they lost faith and recovered when they regained their religious outlook. Sigmund Freud and Albert Ellis maintained that dogmatic religion is significantly correlated with obsessive acts and emotional disturbances. ${ }^{27}$ Yet, I think that for the highly religious Ghanaian cultural context investigation in this area would be enlightening.

Efforts to understand the impact of context-specific variables on subjective well-being will serve greater clinical utility because we can better delineate the when, what, and how to improve people's quality of life. A focus on a systematic investigation of those things that promote mental health and quality of life is important not only because of its primacy in clinical work but also because of the social and economic implications for individuals and society at large.

\section{REFERENCES}

1. Brandtstadter, J., \& Renner, G. Tenacious goal pursuit and flexible goal adjustment: Explication and age-related analysis of assimilative and accommodative strategies of coping. Psychology and Aging 1990;5: 58-67.

2. Menec, V.H., Chiperfield, J.G. The interactive effect of perceived control and functional status on health and mortality among the young-old and oldold adults. Journal of Gerontology: Psychological Sciences 1997; 52B (3): 113-126.

3. Oettingen, G. Cross-cultural perspectives on selfefficacy. In Bandura, A. (Ed.) Self-efficacy in changing societies (pp. 149-175). 1995 New York: Cambridge University Press.

4. Brandtstadter, J. \& Baltes-Gotz, B. Personal control over development and quality of life perspective in adulthood. In P. B. Baltes \& M. M. Baltes (Eds.) Successful Aging: Perspectives from Behavioral Sciences, (pp 197-224).1990 New York: Cambridge University Press.

5. Steptoe, A., Evans, O., \& Fieldman, G. Perceived control over work: Psychophysiological response to self-paced and externally-paced tasks in an adult population sample. International Journal of Psychophysiology 1997; 25: 211-220

6. Fontaine, K.R., McKenna, L., Cheskin, L. J. (1997). Support group membership and perceptions control over health in HIV positive men. Journal of Clinical Psychology 1997; 53: 249-252.

7. Newsom, J.T., Knapp, J.E., \& Schulz, R. Longitudinal analysis of specific domains of internal control and depressive symptoms in patients with recurrent cancer. Health Psychology 1996; 15: 323-331.

8. Wallhagen, M.I. \& Brod, M. Perceived control and well being in Parkinson's disease. Western Journal of Nursing Research 1997; 19: 11-31.

9. Kim, S. L., Sandler, I. N. \& Tein, J. Locus of control as a stress moderator and mediator in children of divorce. Journal of Abnormal Child Psychology 1997; 25: 145-155.

10. Peacock, E. J. \& Wong, P. T. P. Anticipatory stress: The relation of locus of control, optimism, and control appraisals to coping. Journal of Research in Personality 1996; 30: 204-222. 
11. Grob, A. Perceived control and subjective wellbeing across nations and across the life-span. In E.Diener \& E.M.Suh (Eds.), Culture and subjective well-being (pp. 319-339). 2000 Massachusetts: The Massachusetts Institute of Technology Press.

12. Diener, E. Subjective well-being. Journal of American Psychological Association 2000; 55:3543.

13. Diener, E., Suh, E. \& Oishi, S. Recent findings on subjective well-being. Indian Journal of Clinical Psychology 1997; 2: 1-17.

14. Diener, E., Emmons, R. A., Larsen, R. J., \& Griffin, S. The Satisfaction with Life Scale. Journal of Personality Assessment 1985; 49: 7175.

15. Ryff, C. D. \& Keyes, C. L. M. The structure of psychological well-being revisited. Journal of Personal and Social Psychology 1995; 69: 719727

16. Ryff, C. D. Happiness is everything, or is it? Explorations on the meaning of psychological well-being. Journal of Personality and Social Psychology 1989; 57: 1069-1081.

17. Abramson, L. Y., Seligman, M. E. P., and Teasdale, J. D. Learned helplessness in humans: Critique and reformulation. Journal of Abnormal Psychology 1978; 87: 49-74.

18. Heckhausen, J., \& Schulz, R. A lifespan theory of control. Psychological Review 1995; 102: 284304.

19. Ryff, C. D. Psychological well-being in adult life. Current Directions in Psychological Science 1994; 1: 1-3.

20. Peng, Y. Primary and Secondary control in American and Chinese-American adults: crosscultural and life-span development perspectives (Doctoral dissertation, Brandeis University, 1993). Dissertation Abstracts International, 55, 3037.

21. Wrosch, C., Heckhausen, J., \& Lachman, M. E. Primary and secondary control strategies for managing health and financial stress across adulthood. Psychology and Aging 2000; 15: 387399.

\section{APPENDIX A: RYFF'S WELL-BEING SCALE}

When I look at the story of my life, I am pleased about how things have turned out.

I enjoy making plans for the future and working to make them a reality.
22. Diener, E., Oishi, S. \& Lucas, R. E. (2003). Personality, culture, and subjective well-being: Emotional and cognitive evaluations of life. Annual Review of Psychology 2003; 54:403-425.

23. Eckersley, R. Science and culture: changing how we see the world and our place in it. Manning Clark House Inc. Papers (pp.1-5). 2003 Canberra, Australia.

24. Triandis, H.C. Cultural syndromes and subjective well-being. In E.Diener \& E.M.Suh (Eds.), Culture and subjective well-being (pp. 13-36). 2000 Massachusetts: The Massachusetts Institute of Technology Press

25. Diener, E. \& Suh, E. M. Measuring subjective well-being to compare the quality of life of cultures. In E.Diener \& E.M.Suh (Eds.), Culture and subjective well-being (pp. 3-12). 2000 Massachusetts: The Massachusetts Institute of Technology Press.

26. Chou, K. L. Social support and subjective wellbeing among Hong Kong Chinese young adults. Journal of Genetic Psychology 1999; 160: 319331.

27. Hughes, P., Castle, K. \& Bellamy, J. Wellbeing and security study. Anglicare Sydney NCLS Research Paper 6. 2004 Edith Cowan University \& Deakin University.

28. Jones, T., Rapport, L., Hanks, R., Litchtenberg, P. $\&$ Telmet, K. Cognitive and psychosocial predictors of subjective well-being in urban older adults. Clinical Neuropsychology 2003; 17: 3-18.

29. Savelkoul, M., Post M.W., Witte, L.P. \& Borne, H.B. Social support, coping and subjective wellbeing in patients with rheumatic diseases. Patient Education and Counseling 2000; 39: 205-218.

30. Taylor, R., Chatters, L., Hardison, C. \& Riley, A. Informal social support networks and subjective well-being. Journal of Black Psychology 2001; 27:439-463.

31. Makino, Y. \& Tagami, F. Subjective well-being and self-acceptance. Shinrigaku Kenkyu 1998; 69: 143-148.
I think it is important to have new experiences that challenge how you think about yourself and the world.

I tend to be influenced by people with strong opinions.

I judge myself by what I think is important, not by the values of what others think is important.

I have confidence in my own opinions, even if they are contrary to the general consensus. 
When I think about it, I haven't really improved much as a person over the years.

The demands of everyday life often get me down.

In general, I feel I am in charge of the situation in which I live.

I feel like I get a lot out of my friendships

I have not experienced many warm and trusting relationships with others

Maintaining close relationships has been difficult and frustrating for me.

I have a sense of direction and purpose in life.

I don't have a good sense of what it is I am trying to accomplish in life.

For me, life has been a continuous process of learning, changing and growth

I am quite good at managing the many responsibilities of my daily life.

Many days I wake up feeling discouraged about how I have lived my life.

In many ways I feel disappointed about my achievements in life.

* Items are rated on a 5-point scale ranging from Strongly Disagree (1) to Strongly Agree (5)

\section{APPENDIX B: PRIMARY CONTROL ITEMS}

When things don't go according to my plans, my motto is "where there is a will, there is a way". I keep trying until I find a way to make it work.

When faced with a bad situation, I do what I can to change it for the better.
Even when I feel I have too much to do, I find a way to get it all done.

I rarely give up something I am doing, even when things get tough.

When I am overloaded, I find other people to help me get things done.

I would use any means (like support groups, taking special classes, medication) to help me do the things I want to do in life.

When things are not going my way, I find it helpful to get reassurance from others to keep working on it.

If necessary, I would use assistance from my family and friends to achieve my goals.

* On Perceptions of Control, Items are rated on a 7poing scale ranging from Very Bad (1) to Very Good (7). Similarly on Importance of Control or Value, items are rated on No! Not Very Important (1) to Yes! Very Important (7).

\section{APPENDIX C: THE SATISFACTION WITH LIFE SCALE}

In most ways my life is close to my ideal

The conditions of my life are excellent.

I am satisfied with my life.

So far I have gotten the important things I want in life

If I could live my life over, I would change almost nothing.

* Items are rated on a 7-point scale ranging from Strongly Disagree (1) to Strongly Agree (7). 